\title{
Evolución de los patrones de localización de las inversiones industriales en Andalucía
}

\author{
Daniel Coq Huelva*
}

\begin{abstract}
This article analyses the transformations of the industrial activity localisation in Andalucia in the decades of 1980 and 1990. To this end, we use investment data from the industrial registry. The aim is to analyse the spatial localisation patterns of investment and their relationship to the sectorial specialisation of the different territories of the region.

There are specific places that absorb the majority of the available industrial investment, mainly the most important cities (Seville and its metropolitan area in particular) and some locations of the Andalucian territory (e.g. Polo Quimico de Huelva located in the municipality of Palos de la Frontera, or Polo Petroquímico de Algeciras). However, we point out that in the period we have analysed, the Andalucian industry has suffered some transformations.
\end{abstract}

Keywords: industrial localisation, regional development, economic geography, industrial geography, economic re-structuring.

\section{Resumen}

El presente texto analiza las transformaciones en la localización de las actividades industriales en Andalucía en las décadas de 1980 y 1990. Para ello utiliza datos de inversión procedentes del registro industrial. El objetivo es examinar los patrones de localización espacial de las inversiones poniéndolos en relación con la especialización sectorial de los distintos territorios que componen la región.

Son determinados espacios, fundamentalmente las ciudades más importantes (Sevilla y su área metropolitana específicamente) y algunos puntos concretos del territorio andaluz (Polo Químico de Huelva -situado en el término municipal de Palos de la Frontera- o Polo Petroquímico de Algeciras) los que en mayor medida absorben la inversión industrial. No obstante, en el periodo analizado la industria andaluza ha experimentado algunas transformaciones.

Palabras clave: localización industrial, desarrollo regional, geografía económica, geografía industrial, reestructuración económica.

\footnotetext{
*Departamento de Economía Aplicada II, Universidad de Sevilla. Correo-e: dcoq@us.es.
} 


\section{Introducción}

En la mayoría de las economías regionales, la industria suele jugar un papel destacado. La actividad industrial es básica al articular los distintos sectores, no sólo influye directamente en la generación de riqueza, sino también indirectamente, dadas las repercusiones que su funcionamiento tiene sobre el conjunto del tejido productivo. Un elemento esencial en el funcionamiento de la actividad industrial es su distribución espacial. El tipo de distribución espacial dominante se relaciona con los sectores dominantes dentro del conjunto de la actividad industrial de una región, con los cuerpos sociales que sostienen estas actividades e, incluso, con los recursos naturales sobre los que, en ocasiones, se basa su actividad (Polèse, 1994). Pero al igual que otros elementos, la localización de la industria sufre importantes modificaciones con el paso del tiempo (Aurioles y Cuadrado, 1989).

El objetivo de este artículo es analizar la localización de las inversiones industriales en un espacio concreto, en Andalucía; para ello se distinguen dos periodos, las décadas de los ochenta y los noventa.

\begin{tabular}{lr}
\multicolumn{2}{c}{ Cuadro 1 } \\
\multicolumn{2}{c}{ Presentación sintética de la región } \\
\hline & Andalucía \\
\hline Territorio $\left(\mathrm{km}^{2}\right)$ & 87,602 \\
Población (habitantes) & $7^{\prime} 606,848$ \\
PIв per cápita 2003 (euros) & 13,695 \\
\hline
\end{tabular}

Fuente: Instituto de Estadística de Andalucía (2005).

Andalucía es una región española de más de $87,000 \mathrm{~km}^{2}$ (casi $18 \%$ del total del territorio español) con, aproximadamente, 7’600,000 habitantes (lo que supone también alrededor de 18\%, en concreto $17.8 \%$ del total de la población española en 2003). El actual sistema de organización territorial del Estado español divide el país en 17 comunidades autónomas. Andalucía es una de las de mayor territorio y participación poblacional. Sin embargo, sus niveles de riqueza se sitúan considerablemente por debajo de la media española. En este sentido, el producto interno bruto (РIB) per cápita se situó en 2003 en 13,695 euros, mientras que para el conjunto del país alcanzaba 18,208 euros; es decir, era casi $25 \%$ inferior (véase cuadro 1 y mapa I). Esta situa- 


\section{Mapa I \\ División regional española}

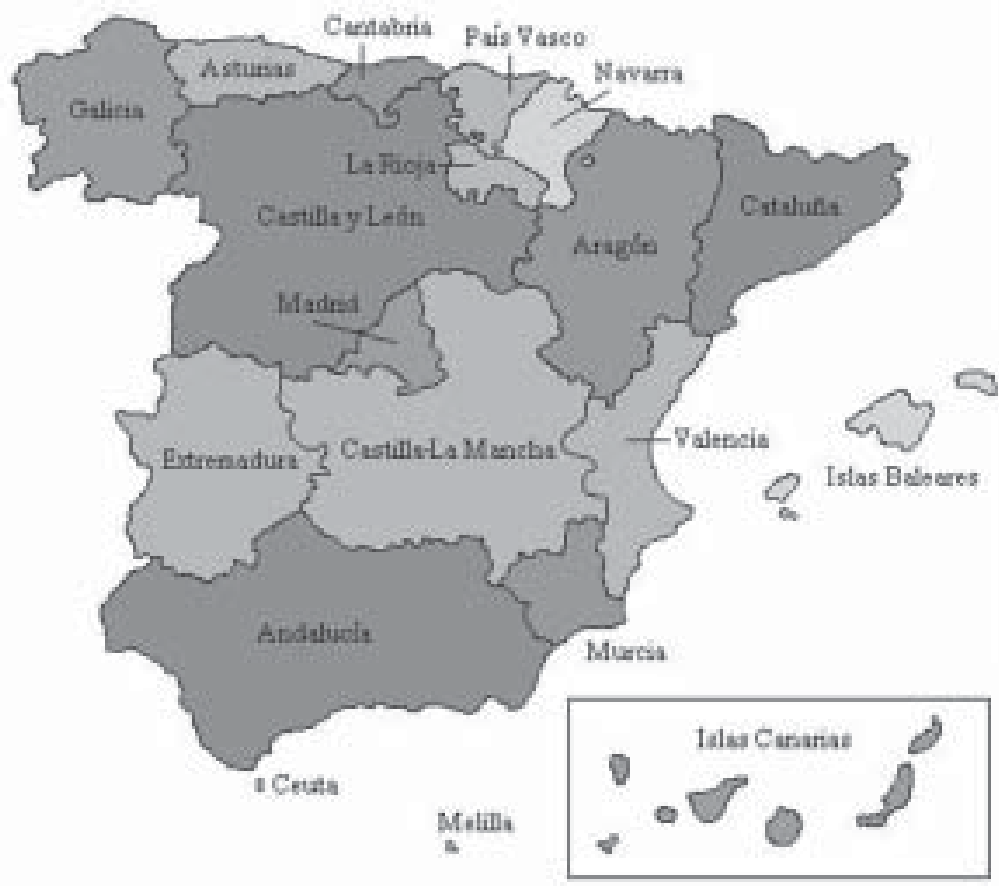

ción explica que la región fuese catalogada como Objetivo 1 en las clasificaciones utilizadas por la Unión Europea, ${ }^{1}$ lo que supuso que fuese considerada de actuación prioritaria por parte de las políticas regionales diseñadas desde Bruselas.

El presente artículo se divide en varios puntos. En primer lugar, se hace una muy somera referencia a las diferentes formas desde las que, con una perspectiva teórica, se ha contemplado el problema de la localización industrial. A continuación, se efectúa una breve revisión de los trabajos que analizan la distribución espacial de las actividades industriales en España en general y en Andalucía en particular. Posteriormente, se observa la distribución de las inversiones industriales en Andalucía durante la década de los ochenta, en concreto en el periodo entre 1984 y 1990. A continuación, se realizará el mismo estudio, pero referido a la evolución durante los noventa, específicamente entre 1991 y 1998. Se insiste, fundamentalmente, en las similitudes y dife-

${ }^{1}$ Una región es considerada Objetivo 1 en el modelo europeo de política regional cuando su renta per cápita no alcanza $65 \%$ de la media de la Unión Europea. 
rencias observadas respecto a la situación en la década anterior, previamente analizada. Finalmente se obtendrán las conclusiones oportunas.

Para realizar la investigación se utilizan esencialmente datos procedentes del Registro Industrial. Se sabe que esta fuente tiene entre sus principales virtudes permitir análisis espaciales muy desagregados, como el que en este artículo pretende realizarse. Pero también es sabido que se trata de un registro que, como tal, no utiliza en el acopio de la información técnicas de muestreo, lo que hace que escape una buena parte de las inversiones realizadas. Con todo, pese a la eventual ausencia de contabilización de ciertas inversiones, consideramos que la fuente estadística empleada puede dar una idea general de la localización espacial de las inversiones en el interior de Andalucía.

\section{La localización desde una perspectiva teórica}

El estudio de los patrones que rigen la localización de las actividades económicas, en general, y la industria, en particular, es un tema clásico en la ciencia regional. Los problemas de la localización han sido abordados desde múltiples perspectivas y esquemas teóricos (Richardson, 1986; Polèse, 1994).

Pese a su heterogeneidad, uno de los elementos comunes de todos los análisis teóricos es su intento por explicar las razones que mueven a la concentración de las actividades económicas en ciertos puntos del espacio, así como las fuerzas que explican este hecho. Esta preocupación se encuentra, por ejemplo, de forma clara en algunos autores de la vieja escuela alemana como A. Weber (1929). También está entre los ortodoxos, aunque más recientes, autores neoclásicos estadounidenses, por ejemplo: L. Moses (1958) o W. Isard (1956).

Otro de los puntos en los que se ha centrado el análisis teórico es la determinación de los factores que promueven la concentración de actividades. En este sentido, desde un primer momento, a muy grandes rasgos, se apuntó la existencia de dos patrones de localización. El primero tendería a concentrar las producciones cerca de los puntos de abastecimiento de materias primas, como el caso de algunas industrias basadas en la transformación de minerales, o incluso el de algunas industrias agroalimentarias, véase la cuestión de la azúcar en Europa. ${ }^{2}$ En otros casos, sin

\footnotetext{
${ }^{2}$ Obtenida a partir del cultivo de remolacha azucarera que implica el transporte de grandes volúmenes.
} 
embargo, la concentración de la producción ocurre cerca de los puntos de demanda final, debido a la actuación de dos elementos fundamentales: la existencia de mano de obra especializada y la presencia de economías de aglomeración (Weber, 1929). Recientemente estos dos elementos -economías de aglomeración y existencia de un mercado de trabajo especializado- fueron retomados por la llamada "nueva geografía económica" (Krugman, 1992).

No obstante, la preocupación por los patrones rectores de la concentración de las industrias no es exclusiva de los autores neoclásicos. Desde otra perspectiva teórica, D. Harvey (1982) explica la localización de las industrias como un elemento que limita los procesos de acumulación de capital, porque lo inmoviliza durante un cierto tiempo, con todo lo que ello implica. Para Harvey, los procesos de acumulación de capital y la inmovilización de las inversiones en determinados espacios actúan como factores que explican la concentración de las inversiones industriales. Dicho en otros términos, las industrias invierten sobre la base de la reinversión de beneficios. Pero estas inversiones están condicionadas por las previamente realizadas, porque buena parte de las plantas y los equipos no pueden trasladarse o, si se trasladan, supone un coste muy importante. De esta forma se tiende a invertir allí donde previamente se ha invertido, y se establece un patrón general de localización espacial caracterizado por la existencia de tendencias centrípetas.

Storper y Walker (1989) insisten en el mismo tipo de argumento, pero consideran que en el caso de nuevas industrias centradas en actividades innovadoras, donde por su 'juventud' se advierten niveles reducidos de acumulación de capital, existen situaciones de 'ventana locacional' que pueden incluir 'nuevos' espacios en la geografía industrial tradicional. Del mismo modo, estos autores exploran otra vía de inclusión de nuevos territorios en las dinámicas de crecimiento industrial a partir de la "expansión hacia la periferia de la industria triunfante”, capaz, por lo tanto, de establecer nuevos espacios industriales sobre la base de la descentralización de actividades vinculadas a los crecimientos de producción.

En todo caso, ya sea por la inmovilización de buena parte del capital o la propia dinámica de implantación de economías de aglomeración en las áreas donde se desarrolla determinado tipo de actividades, la localización industrial cambia lentamente. Sin embargo, el cambio en los patrones de inversión es algo más rápido. Esto ya no es un stock sino un flujo que puede orientarse 
con más facilidad hacia un lugar u otro. Con todo, la localización de las inversiones tampoco es un elemento independiente del patrón de localización industrial general. Suele invertirse en buena medida en plantas y establecimientos ya existentes, con lo que incluso los patrones de inversión dependen fuertemente de la localización previa de los establecimientos productivos.

Por último, si quiere tomarse un indicador algo más dinámico de los cambios en los procesos espaciales, puede analizarse la inversión en nuevas plantas que no depende directamente de inversiones previas, pero que, en todo caso, también será influida por las economías de aglomeración y la existencia de mercados laborales cualificados.

\section{Los estudios sobre localización industrial en Andalucía}

En general, puede afirmarse que la localización es un tema con una sólida implantación teórica dentro de la ciencia regional española. Sin embargo, los análisis empíricos son algo más escasos. Pese a que se han realizado estudios profundos sobre la evolución de la localización de las industrias en Andalucía, en muchas ocasiones se han espaciado considerablemente en el tiempo. Los principales hitos en el análisis de los cambios en la localización espacial de las actividades productivas en Andalucía son los siguientes.

En primer lugar, habría que destacar el trabajo de Rodríguez Sánchez de Alva (1980), donde sobre la base de la teoría neoclásica de la localización se analiza la distribución de los establecimientos industriales en el caso español. Las distancias a los mercados finales y respecto a los lugares de aprovisionamiento de las materias primas eran los principales factores de localización introducidos en dicha aproximación.

El estudio más completo acerca de la localización de las actividades industriales en España, en general, donde simultáneamente se analiza con detalle el caso andaluz, es posiblemente el realizado por Aurioles y Cuadrado (1989). En ese trabajo, se utiliza una aproximación un tanto ecléctica. Por un lado, no se renuncia a aplicar la teoría neoclásica de la localización en la explicación de los patrones de distribución espacial de las actividades. Pero, por otra parte, se reconoce que su capacidad explicativa es reducida. Por ello tratan de identificarse los factores de localización dominantes a partir de la utilización de una metodología basada en la realización de cuestionarios. Los resultados 
son, en todo caso, poco concluyentes a la hora de aislar los factores de localización dominantes en los distintos entornos. El trabajo de Aurioles y Pajuelo (1988) va en la misma línea, pero la identificación de los factores locacionales es más clara.

Desde una lógica mucho más aplicada, Caravaca (1988) analiza la distribución espacial de la industria en el interior de la región; la pone en relación no con factores abstractos de localización sino con las trayectorias de transformación y cambio estructural experimentadas. En este sentido, podría afirmarse que más que los patrones de localización industrial, analiza los modelos de "industrialización geográfica" dominantes en el sector industrial andaluz.

Un esquema parecido siguen Delgado y Román (1995), sólo que, en este caso, junto al cambio estructural de la economía andaluza, se añade el efecto que sobre ella tuvieron los procesos de reestructuración productiva y globalización observables desde al menos 1975. Del mismo modo, estos autores no analizan el sector industrial en su conjunto, sino exclusivamente la principal especialización industrial de la economía andaluza, la agroalimentaria, que por sí sola supone 8,393 de los 39,584 establecimientos manufactureros de la región. ${ }^{3}$ Del mismo modo, su análisis se centra en el examen de la distribución espacial de las inversiones.

Por último, Coq (2001) profundiza en el estudio anterior, analiza detalladamente las distintas dinámicas de acumulación y la estructura de mercados y movimientos corporativos existentes en cada una de las ramas que integra la agroindustria andaluza. De este modo, se llega a una mayor comprensión de la complejidad de los mecanismos que interactúan a la hora de proyectar las dinámicas productivas sobre el territorio de la región.

El presente texto analiza, preferentemente, los cambios en la distribución espacial de las inversiones en el periodo 1980-1998. Por la elección de la variable objeto de estudio, se asemeja al trabajo de Delgado y Román (1995), aunque amplía su campo de observación al conjunto del sector industrial. En la visión del problema objeto de estudio, se investigarán, más bien, los patrones de "industrialización geográfica" que los de "localización espacial”. Es decir, la localización se observa como el resultado de procesos complejos de transformación estructural más que como

${ }^{3}$ Datos obtenidos del directorio de establecimientos con actividad económica en Andalucía del 2003. 
el output de la actuación de factores abstractos. En todo caso, por lo limitado del espacio, se expondrán tan sólo los rasgos más generales que explican la distribución espacial de la industria en Andalucía. Un examen más detallado requeriría bajar a una escala subsectorial y municipal de análisis, lo que trasciende por mucho las posibilidades de este texto.

\section{Distribución espacial de las inversiones industriales en Andalucía durante la década de los ochenta}

La evolución de la localización de la industria en Andalucía está relacionada con los patrones históricos de funcionamiento de su economía regional. Tradicionalmente, la base industrial de la región ha sido débil, lo que explica que su peso dentro del total español haya sido modesto. De esta forma, si la participación territorial y poblacional de Andalucía en el total español es de $18 \%$, aproximadamente, la de la industria no llega a alcanzar 9\%. Esto es, se trata de un territorio con una base industrial débil.

Esta debilidad puede explicarse del siguiente modo. Históricamente, el nivel de desarrollo productivo de la economía andaluza fue inferior que el resto de la economía española. El desarrollo del mercado nacional desde finales del xix llevó a una progresiva destrucción del tejido industrial interno, vinculado a las especializaciones productivas tradicionales de la región (en espacial a la agroalimentaria) y que, por lo tanto, tendía a difundirse a lo largo y ancho del territorio (Delgado, 1981; Delgado y Román, 1995).

Por el contrario, sobre todo a partir de los años sesenta, se desarrollaron determinados tipos de especializaciones productivas como consecuencia de las políticas desarrollistas impulsadas. Fruto de ellas fue el desarrollo de especializaciones como la industria química (Polo de Huelva), la petroquímica (campo de Gibraltar), algunas industrias pesadas como la de construcción naval y otras de importancia menor. En este caso, los patrones de localización industrial serán distintos. Siguiendo el modelo del polo de desarrollo de Perroux (1981), estas industrias son conscientemente localizadas en puntos muy concretos del territorio, frecuentemente coincidentes con las grandes áreas urbanas de la región. De este modo, mientras que la industria “endó- 
gena”, ${ }^{4}$ por ejemplo la agroalimentaria, tiene un patrón de localización relativamente disperso, las nuevas especializaciones productivas se concentran en todos los casos en grandes núcleos urbanos. Esto es una constante desde, al menos, la década de los sesenta.

Los patrones de localización de la inversión dependen en buena medida de estos rasgos estructurales. También en buena medida la inversión se localiza donde se ubican las principales instalaciones industriales. Esto es especialmente evidente en las inversiones destinadas a la ampliación de los equipos existentes. Por ejemplo, en los años ochenta tres cuartas partes de la inversión en ampliaciones de industrias se localizan en las ciudades mayores de 50,000 habitantes y en las grandes áreas metropolitanas de la región, cifra muy superior a su participación en la población $(50.5 \%)$ y, por supuesto, a su participación en el territorio de la región (véase cuadro 2 ).

\section{Cuadro 2}

Distribución espacial de las inversiones en ampliación de industrias en Andalucía en el periodo 1984-1990

\begin{tabular}{lcc}
\hline & $\begin{array}{c}\text { Participación en } \\
\text { ampliación de plantas }\end{array}$ & $\begin{array}{c}\text { Participación en } \\
\text { la población total }\end{array}$ \\
\hline Grandes ciudades $^{5}$ & $76.7 \%$ & $50.5 \%$ \\
Ćiudades intermedias $^{6}$ & $11.9 \%$ & $24.1 \%$ \\
Áreas rurales $^{7}$ & $11.4 \%$ & $25.7 \%$ \\
\hline
\end{tabular}

Fuente: Elaboración propia a partir de los datos obtenidos del Registro Industrial.

${ }^{4}$ Esta es una afirmación bastante general que, por lo tanto, hay que tomar con precaución, ya que, por ejemplo, no todas las industrias agroalimentarias pueden catalogarse como actividades arraigadas o endógenas (Coq, 2001).

${ }^{5}$ Se han considerado urbanos aquellos municipios situados por encima de los 50,000 habitantes en 1981. Esto incluía a las ocho capitales de provincias, así como a los municipios de Algeciras, Marbella y Linares. Adicionalmente, teniendo presente la existencia de un cierto fenómeno de metropolización, se han considerado, en el caso de Sevilla y Cádiz, los municipios que de un modo más claro, desde una perspectiva industrial, se incluyen en el área metropolitana de estas ciudades; es decir, Alcalá de Guadaira, Dos Hermanas, La Rinconada, San Juan de Aznalfarache y Mairena del Aljarafe en el caso de Sevilla, y Chiclana de la Frontera, El Puerto de Santa María, Jerez de la Frontera, San Fernando y Sanlúcar de Barrameda en Cádiz.

${ }^{6}$ Se consideran ciudades intermedias aquellas cuyos municipios oscilan entre los 10,000 y los 50,000 habitantes. Se trata de poblaciones que por su número de habitantes pueden considerarse urbanas, pero que, en el caso concreto de Andalucía, se caracterizan por la importancia que todavía tiene la actividad agraria. Por ello, suelen recibir el calificativo de "ciudades intermedias" o "agrociudades", que resalta el carácter mixto de estas áreas.

${ }^{7}$ Se consideran rurales aquellos municipios con menos de 10,000 habitantes. 
Ello se debe fundamentalmente a la existencia de una fuerte 'inercia locacional' que se justificaría de la siguiente forma: inicialmente, buena parte de las localizaciones industriales fueron el resultado de decisiones 'políticas' tendentes a distribuir la actividad en el territorio. En este sentido, como se ha indicado, se conformaron polos y centros de acumulación. Una vez establecidas, las empresas acumularon ventajas competitivas, inmovilizaron inversiones, generaron un mercado de trabajo relativamente cualificado etc., lo que propició un incentivo para que las nuevas inversiones se concentrasen en los mismos lugares donde lo habían hecho las anteriores.

Este proceso puede verse interrumpido por la aparición de 'ventanas locacionales', es decir, de nuevas actividades en sectores con niveles de acumulación reducidos que presenten mercados que se expandan rápidamente. En el caso de Andalucía, este proceso en general no sucede, lo que explica el mantenimiento de la estructura productiva y el patrón de localización previamente existente.

Dentro de las grandes áreas urbanas, por orden de importancia, la principal receptora de inversiones destinadas a la ampliación de la capacidad productiva es, durante los ochenta, el campo de Gibraltar, donde se localiza uno de los polos de crecimiento promovidos en los años ochenta, especializado en actividades petroquímicas. Ello habla de la importancia que durante este periodo tuvo la ampliación de las actividades del polo petroquímico de Algeciras, así como de la relevancia de otras empresas como Acerinox (que fabrica acero inoxidable, y en la actualidad es una de las empresas mundiales líderes en esta rama) allí instaladas. Le siguen en importancia inversora los principales centros urbano-industriales de la región, como Sevilla, Málaga, Huelva o Cádiz. Se trata por lo tanto de un patrón de localización de las inversiones extremadamente concentrado.

La alta concentración de las inversiones en ampliación de plantas puede observarse más claramente en el mapa II y en el cuadro 3. Ahí puede verse cómo los únicos municipios que concentran más de 5\% de la inversión total en la expansión de plantas ya existentes son Sevilla, Málaga, Los Barrios y San Roque. Se pone, por lo tanto, de manifiesto la alta concentración de las inversiones en áreas muy concretas. Por ejemplo, dos de los municipios del campo de Gibraltar (Los Barrios y San Roque) concentraban más de 30\% de la inversión total (véase cuadro 4). Además destacan los casos de algunos municipios del área metropolitana de 
Sevilla, como Alcalá de Guadaira y Dos Hermanas con participaciones menores, pero en todo caso significativas.

\section{Mapa II}

Distribución espacial por municipios de las inversiones industriales destinadas a la ampliación de plantas en Andalucía en el periodo 1984-1990

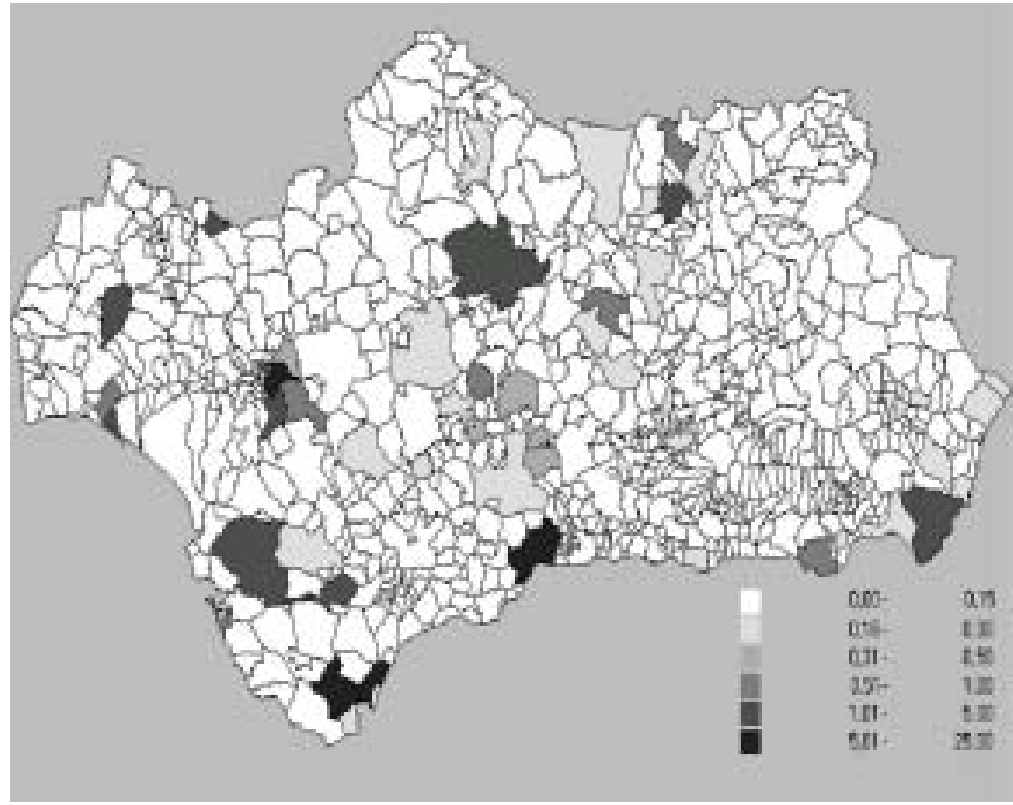

Fuente: Elaboración propia a partir de los datos obtenidos del Registro Industrial.

Por otro lado, los municipios rurales y las ciudades intermedias no absorben flujos inversores de importancia, pese a que, sobre todo en los años ochenta, la importancia de las actividades industriales 'endógenas' preferentemente localizadas en su interior era muy considerable. Las actividades industriales represen$\tan 40.6 \%$ del valor añadido bruto industrial. ${ }^{8}$ Por lo tanto, en Andalucía la industria endógena localizada, en buena medida, en áreas rurales y ciudades interiores no es capaz de seguir el ritmo

${ }^{8}$ Esta cifra es, en todo caso, una aproximación, en la que se han considerado como endógenas las industrias agroalimentarias y ligeras (vestido, calzado, muebles, etc.) y exógenas todas las demás (maquinaria, equipos, industrias extractivas y pesadas, etc.). Se trata de un dibujo que, aunque real, está hecho con un trazo grueso ya que dentro de las industrias extractivas, por ejemplo, hay actividades vinculadas con la base industrial tradicional de la región y, dentro de la agroalimentaria, actividades como la azúcar donde domina la lógica exógena. Los datos han sido tomados de la tabla insumo producto regional de 1980 . 
Cuadro 3

Distribución espacial por municipios de la inversión en ampliación de industrias en Andalucía en el periodo 1984-1990

\begin{tabular}{lc}
\hline & Participación en el total \\
\hline Barrios, Los & $19.4 \%$ \\
San Roque & $17.3 \%$ \\
Málaga & $11.4 \%$ \\
Sevilla & $10.0 \%$ \\
Palos de la Frontera & $3.3 \%$ \\
Dos Hermanas & $2.6 \%$ \\
Córdoba & $2.5 \%$ \\
Níjar & $2.5 \%$ \\
Linares & $2.3 \%$ \\
Jerez de la Frontera & $1.9 \%$ \\
Calañas & $1.6 \%$ \\
Cala & $1.4 \%$ \\
San Fernando & $1.0 \%$ \\
Ejido, El & $1.0 \%$ \\
Alcalá de Guadaira & $0.9 \%$ \\
Huelva & $0.9 \%$ \\
Carolina, La & $0.9 \%$ \\
Martos & $0.8 \%$ \\
San Juan de Aznalfarache & $0.8 \%$ \\
Puente Genil & $0.7 \%$ \\
Roda de Andalucía, La & $0.5 \%$ \\
Lucena & $0.5 \%$ \\
Minas de Riotinto & $0.5 \%$ \\
\hline
\end{tabular}

Fuente: Elaboración propia a partir de los datos obtenidos del Registro Industrial.

inversor de las grandes industrias ubicadas en los centros urbanos. En los ochenta existieron, como más adelante se argumentará con mayor detalle, incipientes sistemas productivos locales situados en entornos rurales y ciudades intermedias. Sin embargo, la ampliación de plantas era un fenómeno poco frecuente a la altura de los años ochenta. Únicamente en casos muy concretos, como Lucena, La Roda de Andalucía, Martos o La Carolina, pueden observarse flujos inversores de una cierta importancia.

En algunos de estos casos -Lucena, por ejemplo- en los ochenta se producen fenómenos claros de industrialización en forma de concentraciones territorializadas de pequeñas y medianas industrias. No obstante, hay que utilizar con mucha precaución estos términos, ya que si bien existen concentraciones de pequeñas empresas, es bastante discutible que las relaciones estableci- 
das entre ellas respondan a los postulados teóricos habitualmente asumidos (Becattini y Rullani, 1995). En otras ocasiones, la relevancia de ciertos municipios puede explicarse por la localización en ellos de grandes industrias modernas que actuarían en forma de enclaves capaces de atraer importantes flujos inversores.

\section{Cuadro 4}

Las inversiones en ampliación de plantas en las grandes áreas urbanas en Andalucía en el periodo 1984-1990

\begin{tabular}{lc}
\hline & $\begin{array}{c}\text { Participación en ampliación } \\
\text { de plantas }\end{array}$ \\
\hline Campo de Gibraltar & $37.3 \%$ \\
Área Metropolitana de Sevilla & $14.7 \%$ \\
Área Metropolitana de Málaga & $11.4 \%$ \\
Área Metropolitana de Huelva & $4.2 \%$ \\
Área Metropolitana de Cádiz & $3.3 \%$ \\
\hline
\end{tabular}

Fuente: Elaboración propia a partir de los datos obtenidos del Registro Industrial.

Si las inversiones en ampliación de plantas dependen de dónde se encuentren previamente instaladas y tienden a reproducir las dinámicas locacionales históricamente dominantes, no ocurre lo mismo con la inversión en nuevas industrias donde existe también un componente histórico que explica la evolución de este agregado, aunque su capacidad explicativa es menor.

\section{Cuadro 5}

Distribución espacial de las inversiones en nuevas industrias en Andalucía en el periodo 1984-1990

\begin{tabular}{lcc}
\hline & $\begin{array}{c}\text { Participación en } \\
\text { nuevas inversiones }\end{array}$ & $\begin{array}{c}\text { Participación en la } \\
\text { población total }\end{array}$ \\
\hline Grandes ciudades & $45.3 \%$ & $50.5 \%$ \\
Ciudades intermedias & $23.1 \%$ & $24.1 \%$ \\
Áreas rurales & $32.5 \%$ & $25.7 \%$ \\
\hline
\end{tabular}

Fuente: Elaboración propia a partir de los datos obtenidos del Registro Industrial.

Como puede verse en el cuadro 5, la participación de las grandes ciudades y sus áreas metropolitanas en las inversiones en nuevas plantas era de $45.3 \%$, cifra muy inferior al 76\% contabilizado para la ampliación de plantas ya existentes. De la misma forma, la participación de las áreas rurales (32.5\%) era muy supe- 
rior para el caso de inversiones en ampliación (11.4\%). Por lo tanto, desde esta perspectiva puede hablarse de la existencia de fenómenos muy incipientes de descentralización productiva en la Andalucía de los ochenta, sobre todo, con base en la apertura de nuevos establecimientos.

Estos fenómenos eran durante los ochenta bastante generales en la economía española; se observaban a lo largo de toda su geografía procesos incipientes de descentralización productiva y desarrollo industrial en áreas rurales que finalmente, en su mayoría, no terminaron por cuajar (Méndez et al., 1999). En el mapa III y en el cuadro 6 pueden verse, por un lado, el fuerte dominio de las principales áreas metropolitanas y, por otro, las zonas rurales y ciudades intermedias con una cierta capacidad de generación de actividades industriales.

\section{Mapa III}

Distribución espacial por municipios de las inversiones industriales destinadas a la construcción de nuevas plantas en Andalucía en el periodo 1984-1990

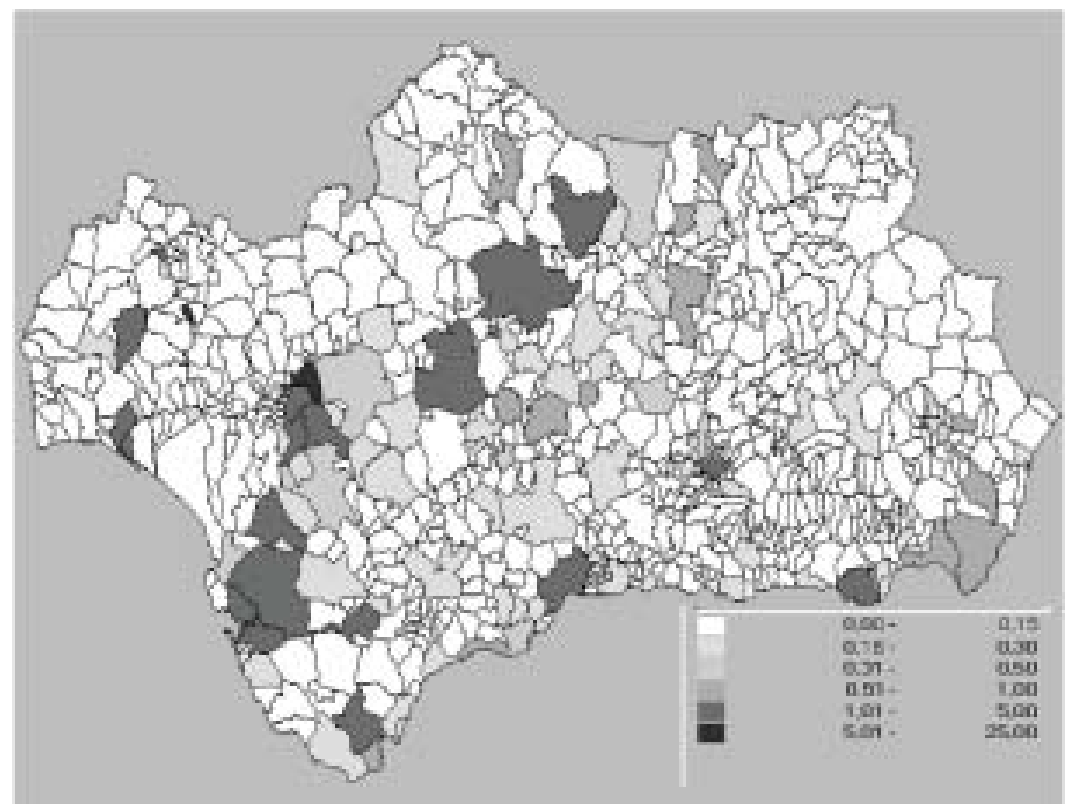

Fuente: Elaboración propia a partir de los datos obtenidos del Registro Industrial.

Por último, si se agregan las inversiones en ampliación con las de nueva planta, se obtiene la inversión total. Las primeras representan $52.5 \%$ de la inversión total frente a $47.5 \%$ de las se- 


\section{Cuadro 6}

Distribución espacial por municipios de las inversiones industriales destinadas a la construcción de nuevas plantas en Andalucía en el periodo 1984-1990

\begin{tabular}{|c|c|}
\hline & Participación en el total \\
\hline Minas de Riotinto & $8.5 \%$ \\
\hline Rinconada, La & $6.3 \%$ \\
\hline Palos de la Frontera & $5.0 \%$ \\
\hline Sevilla, & $4.1 \%$ \\
\hline Barrios, Los & $3.6 \%$ \\
\hline Puerto de Santa María, El & $3.4 \%$ \\
\hline Granada & $3.2 \%$ \\
\hline Málaga & $2.9 \%$ \\
\hline Alcalá de Guadaira & $2.9 \%$ \\
\hline Dos Hermanas & $2.2 \%$ \\
\hline Huelva & $2.2 \%$ \\
\hline Calañas & $2.0 \%$ \\
\hline Montoro & $1.9 \%$ \\
\hline Lebrija & $1.6 \%$ \\
\hline Ejido, El & $1.6 \%$ \\
\hline Puerto Real & $1.5 \%$ \\
\hline Córdoba & $1.2 \%$ \\
\hline Jerez de la Frontera & $1.2 \%$ \\
\hline Jabugo & $1.1 \%$ \\
\hline Écija & $1.1 \%$ \\
\hline Brenes & $0.9 \%$ \\
\hline Níjar & $0.8 \%$ \\
\hline Jaén & $0.8 \%$ \\
\hline Alhendín & $0.7 \%$ \\
\hline Mojonera, La & $0.7 \%$ \\
\hline Puente Genil & $0.7 \%$ \\
\hline Carcabuey & $0.7 \%$ \\
\hline Pozoblanco & $0.7 \%$ \\
\hline Sorbas & $0.6 \%$ \\
\hline Almería & $0.6 \%$ \\
\hline Algeciras & $0.6 \%$ \\
\hline Bailén & $0.6 \%$ \\
\hline Carolina, La & $0.6 \%$ \\
\hline Lucena & $0.6 \%$ \\
\hline Cantoria & $0.6 \%$ \\
\hline Marbella & $0.6 \%$ \\
\hline
\end{tabular}

Fuente: Elaboración propia a partir de los datos obtenidos del Registro Industrial. 
gundas. Es decir, la participación de cada una de las dos categorías es muy semejante. De esta forma, la distribución de las inversiones totales aparece como muestra el cuadro 7.

\section{Cuadro 7}

Distribución espacial por municipios de la inversión industrial total en Andalucía en el periodo 1984-1990

\begin{tabular}{lcc}
\hline & $\begin{array}{c}\text { Participación en } \\
\text { total de inversiones }\end{array}$ & $\begin{array}{c}\text { Participación en la } \\
\text { población total }\end{array}$ \\
\hline Grandes ciudades & $61.8 \%$ & $50.5 \%$ \\
Ciudades intermedias & $16.8 \%$ & $24.1 \%$ \\
Áreas rurales & $21.4 \%$ & $25.7 \%$ \\
\hline
\end{tabular}

Fuente: Elaboración propia a partir de los datos obtenidos del Registro Industrial.

Como puede observarse en el cuadro 7, el peso de las grandes ciudades sigue siendo dominante (61.8\%). Por lo tanto, en términos generales absorbe seis de cada 10 euros invertidos.

\section{Mapa IV}

Distribución espacial por municipios de las inversiones industriales totales en Andalucía en el periodo 1984-1990

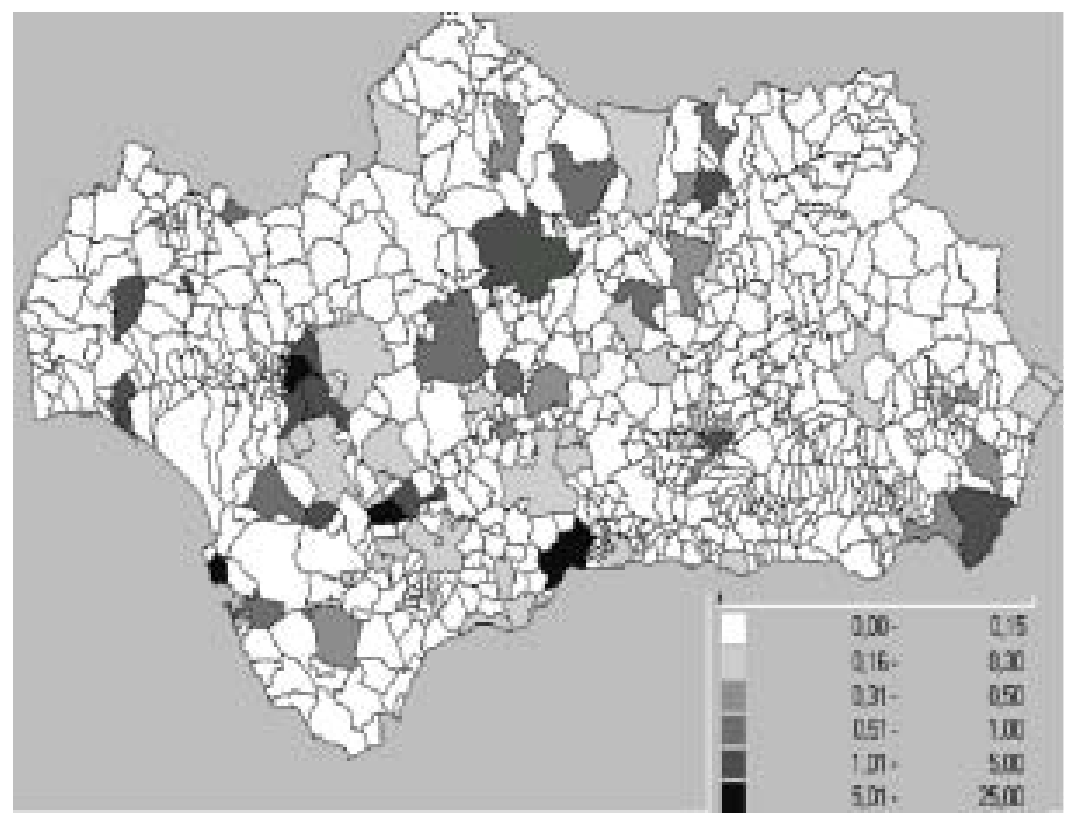

Fuente: Elaboración propia a partir de los datos obtenidos del Registro Industrial. 


\section{Cuadro 8}

Distribución espacial por municipios de las inversiones industriales totales en Andalucía en el periodo 1984-1990

\begin{tabular}{lc}
\hline & Participación en el total \\
\hline Barrios, Los & $12.0 \%$ \\
San Roque & $9.2 \%$ \\
Málaga & $7.4 \%$ \\
Sevilla & $7.2 \%$ \\
Minas de Riotinto & $4.2 \%$ \\
Palos de la Frontera & $4.1 \%$ \\
Rinconada, La & $3.2 \%$ \\
Dos Hermanas & $2.4 \%$ \\
Córdoba & $1.9 \%$ \\
Alcalá de Guadaira & $1.8 \%$ \\
Calañas & $1.8 \%$ \\
Níjar & $1.7 \%$ \\
Granada & $1.7 \%$ \\
Puerto de Santa María, El & $1.7 \%$ \\
Jerez de la Frontera & $1.6 \%$ \\
Huelva & $1.5 \%$ \\
Linares & $1.3 \%$ \\
Ejido, El & $1.3 \%$ \\
Montoso & $0.9 \%$ \\
Lebrija & $0.8 \%$ \\
Carolina, La & $0.7 \%$ \\
Cala & $0.7 \%$ \\
Puerto Real & $0.7 \%$ \\
Martos & $0.7 \%$ \\
Saija & $0.6 \%$ \\
\hline
\end{tabular}

Fuente: Elaboración propia a partir de los datos obtenidos del Registro Industrial.

Como puede apreciarse, el mapa Iv (y el cuadro 8 que constituye su referencia cuantitativa), que muestra la distribución espacial de las inversiones totales, se asemeja más al mapa II, el cual revela la distribución de las inversiones destinadas a ampliación, que al mapa III, que exhibe la distribución de las inversiones destinadas a nuevas industrias. Esto tiene el siguiente efecto: pese a que existen incipientes procesos de descentralización productiva, son aún demasiado débiles para cambiar la distribución to- 
tal de las inversiones en la región. Por lo tanto, la inversión sigue guiada por patrones tendentes a su concentración en puntos muy concretos del espacio.

\section{La distribución espacial de las inversiones en Andalucía durante la década de los noventa}

Entre 1980 y 1998, la economía andaluza continuó el proceso de transformación estructural que la caracterizó en las décadas precedentes. El resultado fue la pérdida de empleo en el sector agrario, que disminuyó en más de 16 puntos en apenas 18 años. De la misma forma, la economía andaluza sigue terciarizándose hasta el punto que en 1995 cerca de dos terceras partes dependían del sector servicios.

Frente a ello, la industria no sólo estancó su progreso sino que se redujo ligeramente, perdió 1.7 puntos en el periodo considerado. Es decir, el crecimiento que hubo en estos años, a diferencia del dominante en las décadas de los sesenta y setenta, no tuvo un perfil industrialista. En este contexto es interesante analizar cómo se comporta espacialmente la inversión en el sector (véase cuadro 9).

\section{Cuadro 9}

Distribución sectorial del empleo en Andalucía en 1980 y 1998

\begin{tabular}{lrcr}
\hline & Empleo 1980 & Empleo 1998 & $\begin{array}{r}\text { Diferencia } \\
1980-1998\end{array}$ \\
\hline Agricultura & 28.1 & 12.3 & -15.8 \\
Industria & 15.3 & 13.6 & -1.7 \\
Construcción & 8.6 & 11.0 & 2.4 \\
Servicios & 48.1 & 63.0 & 14.9 \\
\hline
\end{tabular}

Fuente: Instituto de Estadística de Andalucía (1998).

La situación en los años noventa tenía como principal interrogante la evolución de los incipientes procesos de localización de inversiones industriales de nueva planta en algunos entornos rurales y ciudades intermedias que se observa en la década anterior. Ante este hecho cabían dos posibilidades. La primera era que estos procesos se afirmaran definitivamente, lo que significaría que la inversión industrial se localizase en buena medida lejos de los tradicionales centros urbano-industriales para concentrarse en los nuevos espacios valorados por las dinámicas de 
acumulación de capital. Esto podría suceder, sobre todo, para el caso de las inversiones destinadas a ampliación de las plantas fundadas en los años anteriores.

\section{Cuadro 10}

Distribución espacial de las inversiones en ampliación de industrias en Andalucía en el periodo 1984-1998

\begin{tabular}{lcc}
\hline & $\begin{array}{c}\text { Participación en } \\
\text { ampliación de plantas } \\
\text { en los años 90 }\end{array}$ & $\begin{array}{c}\text { Participación en } \\
\text { ampliación de plantas } \\
\text { en los años 80 }\end{array}$ \\
\hline Grandes ciudades & $74.9 \%$ & $76.7 \%$ \\
Ciudades intermedias & $15.7 \%$ & $11.9 \%$ \\
Áreas rurales & $9.4 \%$ & $11.4 \%$ \\
\hline
\end{tabular}

Fuente: Elaboración propia a partir de los datos obtenidos del Registro Industrial.

Como puede observarse en el cuadro 10, esta eventualidad no ocurre y, por ende, desde esta perspectiva la situación es básicamente continuista. La inversión en ampliaciones sigue localizándose en centros urbano-industriales muy concretos, alejados de cualquier espacio rural o cualquier ciudad intermedia. Con todo, la participación de las ciudades interiores creció ligeramente, lo que dio muestras de la posible existencia de una cierta renovada dinámica inversora en ellas. Esto puede verse más claramente si se examina el mapa v sobre localización de las inversiones industriales destinadas a la ampliación de las plantas ya existentes. En él puede observarse cómo la inversión en ampliaciones se concentra en los grandes núcleos urbanos, y la participación de los rurales y las ciudades intermedias es muy reducida. En este sentido, apenas se notan diferencias importantes con el mapa que refleja la distribución espacial de la misma magnitud en la década precedente.

No obstante, también se mantiene la dinámica anterior tendente a que las inversiones en nuevas plantas se concentran en áreas rurales y ciudades intermedias. De hecho, su participación en la inversión total es ligeramente superior a su peso poblacional. Esto indica que se mantienen los procesos de instalación de nuevas empresas y establecimientos productivos en determinados espacios de la región, aunque no se perciba todavía claramente la existencia de inversiones importantes que apunten a la ampliación de los establecimientos previamente implantados. 


\section{Mapa v}

Distribución espacial por municipios de las inversiones industriales destinadas a la ampliación de plantas en Andalucía en el periodo 1990-1998

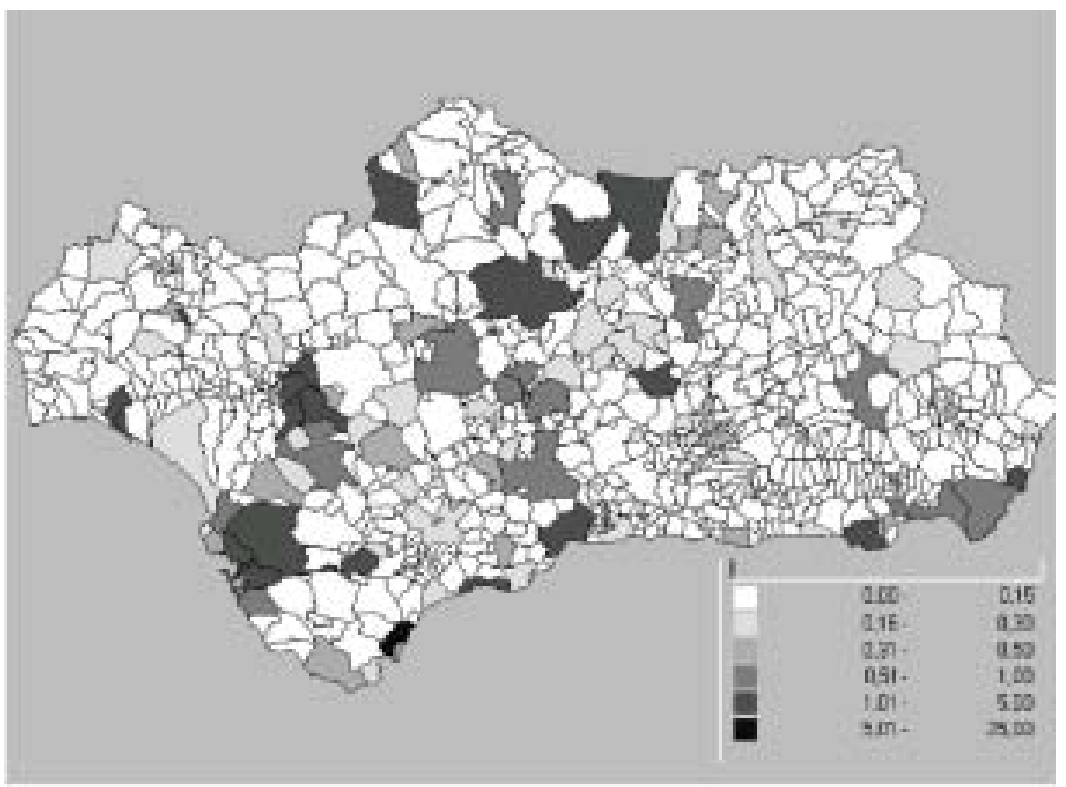

Fuente: Elaboración propia a partir de los datos obtenidos del Registro Industrial.

\section{Cuadro 11}

Distribución espacial de las inversiones en nuevas industrias en Andalucía en el periodo 1984-1998

\begin{tabular}{lcc}
\hline & $\begin{array}{c}\text { Participación en } \\
\text { nuevas inversiones } \\
\text { en los años } 90\end{array}$ & $\begin{array}{c}\text { Participación en } \\
\text { nuevas inversiones } \\
\text { en los años } 80\end{array}$ \\
\hline Grandes ciudades & $43.9 \%$ & $45.3 \%$ \\
Ciudades intermedias & $23.7 \%$ & $23.1 \%$ \\
Áreas rurales & $32.4 \%$ & $32.5 \%$ \\
\hline
\end{tabular}

Fuente: Elaboración propia a partir de los datos obtenidos del Registro Industrial.

De hecho, si se examina esta distribución detenidamente, puede observarse cómo los ejes que concentran la inversión en nuevas plantas en Andalucía aparecen en los noventa algo más claros que en la década precedente. De este modo, puede verse que el eje entre Córdoba y Málaga, y en menor medida la campiña sevillana y la subética cordobesa, son las áreas que en mayor 
medida atraían la inversión industrial. Por lo tanto, parece que las áreas en las cuales acontecen algunos procesos de crecimiento de la inversión están ahora más definidas que antes.

\section{Mapa VI}

Distribución espacial por municipios de las inversiones industriales destinadas a la construcción de nuevas plantas en Andalucía en el periodo 1990-1998

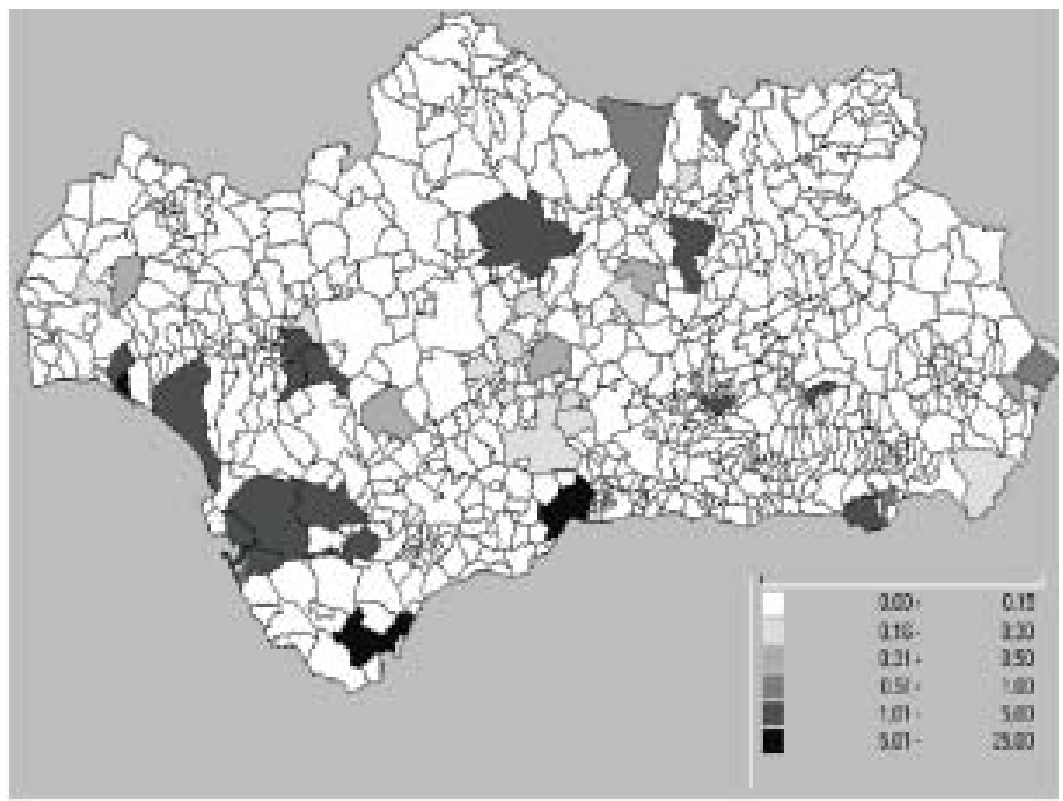

Fuente: Elaboración propia a partir de los datos obtenidos del Registro Industrial.

En realidad, las inversiones en nuevas plantas y en ampliaciones representan aproximadamente 50\% del total. Por ello, en la distribución final de la inversión total vuelven a sobresalir las grandes ciudades, en una situación que esencialmente es también continuista respecto a la anterior. Una representación gráfica de los flujos inversores totales en Andalucía en la década de los noventa vuelve a mostrar cómo la inversión se concentra en los principales núcleos urbanos de la región, como son el Campo de Gibraltar, Huelva, Málaga y las áreas metropolitanas de Sevilla y Cádiz (véase mapa vi y cuadro 11).

En este sentido, se mantiene un patrón de comportamiento observable, al menos desde mediados de los setenta. Es, por así decirlo, el patrón de transformación de las estructuras productivas que emerge tras la crisis del petróleo. Por tanto, no puede 
afirmarse que cambie como consecuencia de la incorporación de España a la entonces llamada Comunidad Económica Europea en 1986, aunque la necesidad de competir en un mercado más amplio y con niveles de eficiencia mayores pudo fortalecer y profundizar las dinámicas ya observables (véase mapa VII).
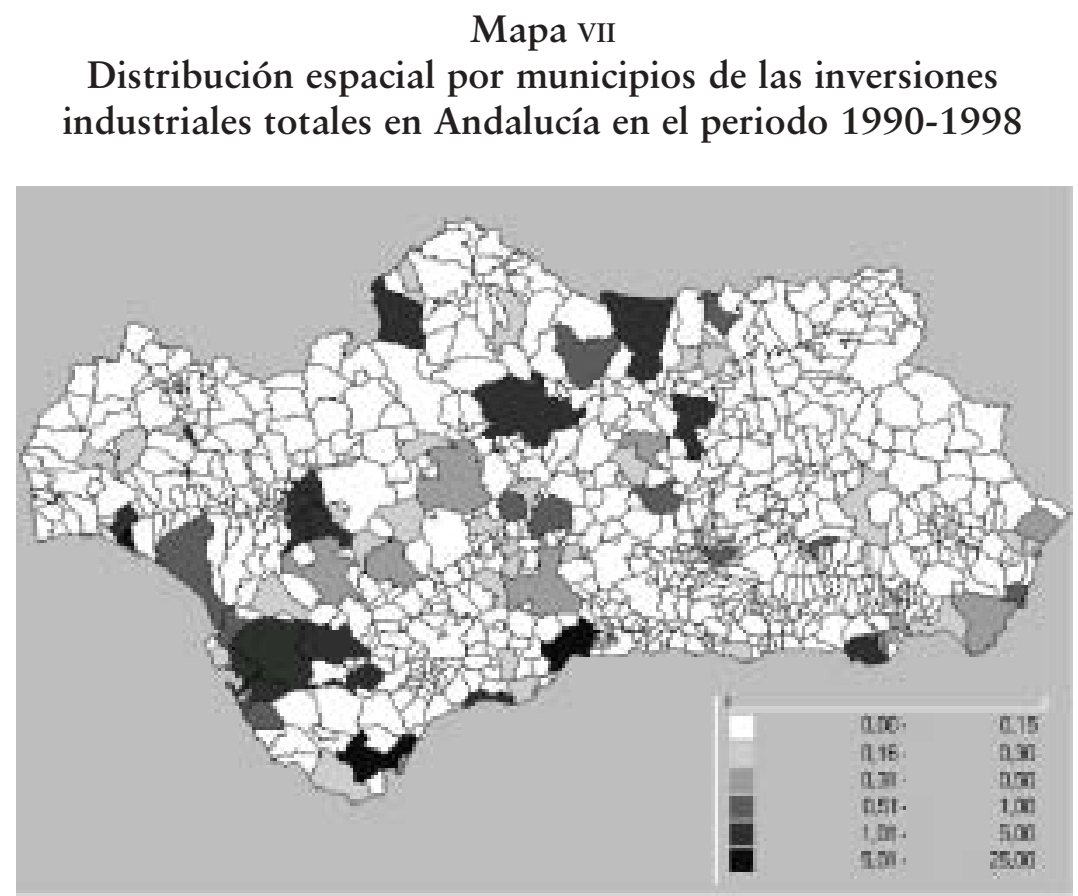

Fuente: Elaboración propia a partir de los datos obtenidos del Registro Industrial.

\section{Conclusiones}

El análisis de los patrones de distribución espacial de la inversión en Andalucía permite obtener varias conclusiones acerca de su evolución. Entre ellas se encuentran, en primer lugar, que la distribución espacial de la inversión refleja los orígenes de la industria andaluza y su relación con el resto del tejido regional. De esta forma, las inversiones se concentran en diversos enclaves que coinciden con algunas de las principales áreas urbanas de la región; estos enclaves mantienen una fuerte dinámica inversora durante todo el periodo estudiado.

Además, la estructura inversora se muestra muy estable a lo largo del tiempo. El predominio de las principales áreas urbanas permanece prácticamente sin cambios durante las dos décadas 
estudiadas. Dentro de ellas, la importancia de áreas como el Campo de Gibraltar, Huelva, Málaga o la zona metropolitana de Sevilla también se sostiene en el tiempo.

Por último, en ciertos momentos se observan, no obstante, algunos procesos incipientes de apertura de nuevas instalaciones productivas en algunos entornos rurales y ciudades intermedias. Se trata de procesos que se mantienen en el tiempo, aunque no son lo suficientemente intensos como para romper la dinámica inversora dominante en la región centrada en la valoración de las actividades situadas en entornos urbanos.

\section{Bibliografía}

Aurioles, Joaquín y Juan Ramón Cuadrado-Roura (1989), La localización industrial en España, Fundación FIEs, Madrid.

y Alfonso Pajuelo (1988), "Factores determinantes de la localización industrial en España”, Papeles de Economía Española, 35: 188-207.

Becattini, Giacomo y Enzo Rullani (1995), "Le District industriel: un concept socioéconomique", en Alain Rallet y André Torre (eds.), Économie industrielle et économie spatiale, Economica, París.

Caravaca, Inmaculada (1988), Industria y territorio en Andalucía, Universidad de Sevilla, Instituto de Desarrollo Regional, Sevilla.

Coq, Daniel (2001), Impactos económicos y territoriales de la reestructuración: la industria agroalimentaria en Andalucía, tesis doctoral, www.eumed.net/tesis_doctorales.

Delgado, Manuel (1981), Marginación y dependencia de la economía andaluza, Monte de Piedad y Caja de Ahorros de Córdoba, Córdoba.

y Carlos Román (1995), "Impactos territoriales de la reestructuración del agroalimentario en Andalucía”, Revista de Estudios Regionales, 42: 83-95.

Harvey, David (1982), The Limits to Capital, Blackwell, Londres. 
Instituto de Estadística de Andalucía (1998), Anuario Estadístico de Andalucía, Instituto de Estadística de Analucía, Consejería de Economía, Junta de Andalucia.

(2005), Anuario Estadístico de Andalucía, Instituto de Estadística de Andalucía, Consejería de Economía, Junta de Andalucia.

Isard, Walter (1956), Location and Space - Economy: A General Theory Relating to Industrial Location, Market Areas, Land Use, Trade, and Urban Structure, MIT Press, Cambridge, Massachusetts.

Krugman, Paul (1992), Geografía y comercio, Antoni Bosch Ed., Barcelona.

Méndez, Ricardo, Juana Rodríguez y Rosa Mecha (1999), “Medios de innovación y desarrollo local en Castilla-La Mancha", Anales de geografía de la Universidad Complutense: 141-167.

Moses, Leon (1958), "Location and the Theory of Production", Quarterly Journal of Economics, 72: 259-272.

Perroux, François (1981), Pour une philosophie du nouveau développement, Aubier, París.

Polèse, Mario (1994), Économie spatiale et urbaine: Logique spatiale des mutations économiques, Economica, París.

Richardson, Harry Ward (1986), Economía regional y urbana, Alianza Editorial, Madrid.

Rodríguez Sánchez de Alva, Alfonso (1980). El suelo como factor de localización industrial: planteamiento general y análisis del caso español, Centro de Estudios y Ordenación del Territorio y el Medio Ambiente, Madrid.

Storper Michael y Richard Walker (1989), The Capitalist Imperative: Territory, Technology and Industrial Growth, Basil Blackwell, Oxford. 
Weber, Alfred (1929), Theory of Location of Industries, University of Chicago Press, Chicago.

Recibido: 6 de octubre de 2004. Reenviado: 28 de abril de 2005. Aceptado: 4 de mayo de 2005.

Daniel Coq Huelva es doctor en ciencias económicas por la Universidad de Sevilla. En esta institución labora actualmente en el Departamento de Economía Aplicada II. Líneas actuales de investigación: economía regional, economía financiera internacional, epistemología de la ciencia económica y economía cuantitativa. Destacan como sus últimas publicaciones: "Acumulación de capital y cambio técnico en el sector de las almazaras", Olivae, revista del Consejo Oleícola Internacional, 2004, pendiente de publicación; "Epistemología, economía y espacio/territorio", Revista de Estudios Regionales, núm. 69, 2003, pp.115-136; "La perspectiva institucionalista de desarrollo regional: Una crítica constructiva", Ekonomiaz, revista vasca de economía, núm. 49, 2002, pp. 238-255, y "Reestructuración y sistemas productivos locales en Andalucía: El caso de la industria agroalimentaria en el eje transversal central norte”, Sociología del Trabajo, núm. 44, 2002, pp. 97-125. 